\title{
Identifying the Potential Barriers to Women Entrepreneurship and Studying their Interrelationships using ISM Methodology
}

\author{
Veena Aggarwal \\ Recventures Education \\ services Private Limited, \\ Delhi, India
}

\author{
Remica Aggarwal \\ School of Business, University \\ of Petroleum \& Energy Studies \\ Dehradun, India
}

\author{
R. Raghunathan \\ Department of Management \\ BITS Pilani, Pilani, India
}

\begin{abstract}
This paper focuses on exploring various barriers towards practicing women entrepreneurship and the interrelationships amongst them through Interpretive Structural Modeling Methodology (ISM). Results suggest that Lack of motivation (LM), Lack of education \& training (LET), Lack of infrastructure (LI), Negative attitude of society (NAS), Lack of knowledge and awareness (LKA), Market issues (MI)falls under high dependence and low to medium driving power are dependent criteria whereas Family problems (FP), Absence of societal concerns (ASC), Financial concerns (FC), Geographical factors (GF), Political factors (PF), Peer group factor (PGF) have low dependence but high driving power and hence they are drivers
\end{abstract}

\section{Keywords}

Women entrepreneurship, Interpretive Structural Modeling Methodology, MIC -Mac Analysis

\section{INTRODUCTION}

The evolution of the Indian entrepreneurship can be traced back to even as early as Rig-Veda, when metal handicrafts existed in the society. Post independence, it is the big business houses of the - Birlas, Bajajs, Goenkas and Mahindras came into existence. By the 2000s, a new breed of entrepreneurs from PVR Cinema's Ajay Bijli to MakeMyTrip's Deep Kalra took wing. In last few years, a new generation of pumped up entrepreneurs and startups has taken centre stage who are tech savvy, deeply networked and collaborative and their ventures have a strong internet flavour. These includes the broader Flipkarts and Snapdeals brands along with the newcomers which provide verticalised online marketplaces for everything from furniture (Urban Ladder, Pepper Fry) to food (Zomato, Foodpanda) to healthcare (Practo, Lybrate). A remarkable exhibit of potential and proficiency shown by these startups has made global investors to venture in the Indian startup ecosystem. This accelerated funding coupled with the innovative and incrementally competent growth of these startups to set as world class companies has set benchmark and hope for many aspiring entrepreneurs.

These startups or entrepreneurship ventures are largely dominated and managed by males. Though a large percentage of women are showing interest towards entrepreneurship, often they are restricted by various causes both on personal as well as professional front which hinders their growth and motivation and left a limited scope of entrepreneurship than their male counterparts. Only those women with strong self esteem and confidence survives amidst the race.

This study aims at understanding perception of beginners on women entrepreneurship and the various barriers towards practicing women entrepreneurship particularly in India. Thereafter the interrelationships amongst these barriers have been studied using the Interpretive Structural Modelling technique (ISM). The paper is organized as follows: Section 2 presents the literature review on entrepreneurship and identification of various barriers towards it. Section 3 presents the ISM methodology in detail. Section 4 presents the case and the ISM model. Finally section 5 presents the conclusions and future directions.

\section{LITERATURE REVIEW}

\subsection{Women Entrepreneurship}

Globally, the phenomenon of women entrepreneurship is flourishing. The on-going development of women entrepreneurship not only acts as anti-poverty instrument in households but also contributes to the personal empowerment. There are several factors such as level of education, occupation and sectoral experience, business expertise, managerial skills, communication and relationship building skills as well as business and management competencies (such as budgeting and managing finances, planning and undertaking business operations, entrepreneurial competencies (innovation, risk taking, creativity, idea generation) and human relations competencies (hiring, staff development, leadership), hard work and perseverance and variety of products offered which contribute as success factors for female entrepreneurship [1]. But at the same time , there are numerous barriers as well which hinders its growth. This paper focuses on identifying the various barriers to successful women entrepreneurship which is discussed in the next sub-section.

\subsection{Identification of barriers to women entrepreneurship}

Though in the last two decades, increasing numbers of Indian women have entered the field of entrepreneurship, not many are successful and this is because of several reasons or causes both at personal as well as professional front. Various barriers have been identified in this section based on the literature review of various articles past 20 years. This has been done by doing google search for keywords such as "barriers to women entrepreneurship", "entrepreneurship barriers" , "factors affecting women entrepreneurship", "barriers to small medium enterprises", "barriers to entrepreneurial growth in India", "women entrepreneurship in India" etc. Searches have been made through Mendeley, Research gate, google search engine etc. The research papers received were explored and around 14 criteria are selected which are further categorise into broader categories of personal - familial; socio-cultural, environmental, political, cultural and technological factors. 


\section{INTERPRETIVE STRUCTURAL MODELLING METHODOLOGY}

Table 1 : Barriers to women entrepreneurship

\begin{tabular}{|c|c|c|c|}
\hline $\begin{array}{l}\text { S. } \\
\text { No. }\end{array}$ & Barriers & Components & Author \\
\hline 1. & $\begin{array}{l}\text { Lack } \\
\text { of } \\
\text { motivation } \\
\text { (LM) }\end{array}$ & $\begin{array}{c}\text { This includes lack of } \\
\text { motivation, lack of self- } \\
\text { confidence, fear of failure, } \\
\text { lack of power on part of } \\
\text { person. }\end{array}$ & [2]- [10] \\
\hline 2. & $\begin{array}{c}\text { Lack } \\
\text { of } \\
\text { Education } \\
\text { (LE) }\end{array}$ & $\begin{array}{l}\text { This comprise of low level } \\
\text { of education and lack of } \\
\text { professional and technical } \\
\text { skills. }\end{array}$ & $\begin{array}{c}\text { [11],[12], } \\
{[13],} \\
{[4],[7],} \\
{[10]}\end{array}$ \\
\hline 3. & $\begin{array}{c}\text { Family } \\
\text { problems } \\
(\mathrm{FP})\end{array}$ & $\begin{array}{l}\text { This includes family } \\
\text { problems, lack of family } \\
\text { support, inability to } \\
\text { maintain balance between } \\
\text { work place and family. }\end{array}$ & $\begin{array}{c}\text { [11], [3], } \\
\text { [7], [9] }\end{array}$ \\
\hline 4. & $\begin{array}{c}\text { Lack } \\
\text { of } \\
\text { infrastructure } \\
\text { (LI) }\end{array}$ & $\begin{array}{l}\text { This may include lack of } \\
\text { infrastructure, lack of } \\
\text { entrepreneurial capacity, } \\
\text { age of enterprise. }\end{array}$ & $\begin{array}{c}6], \quad[7] \\
,[10]\end{array}$ \\
\hline 5. & $\begin{array}{l}\text { Negative } \\
\text { attitude } \\
\text { of society } \\
\text { (NAS) }\end{array}$ & $\begin{array}{l}\text { This includes negative } \\
\text { attitude of authorities and } \\
\text { society towards women. } \\
\text { Also includes gender } \\
\text { stereotypes. }\end{array}$ & $\begin{array}{l}{[14],} \\
{[11],} \\
{[15],} \\
{[10]}\end{array}$ \\
\hline 6. & $\begin{array}{c}\text { Lack } \\
\text { of } \\
\text { knowledge } \\
\text { and } \\
\text { awareness } \\
\text { (LKA) }\end{array}$ & $\begin{array}{c}\text { This includes lack of } \\
\text { awareness towards women } \\
\text { entrepreneurship, lack of } \\
\text { entrepreneurial } \\
\text { management / lack of } \\
\text { information/ lack of market } \\
\text { experience. }\end{array}$ & $\begin{array}{c}{[14],} \\
{[13],} \\
{[5],[7],} \\
{[8],[10]}\end{array}$ \\
\hline 7. & $\begin{array}{l}\text { Absence } \\
\text { of } \\
\text { societal } \\
\text { concerns } \\
\text { (ASC) }\end{array}$ & $\begin{array}{l}\text { This includes lack of } \\
\text { motivation from society for } \\
\text { women entrepreneurship, } \\
\text { lack of co-operation and } \\
\text { absence of supportive } \\
\text { organization. }\end{array}$ & $\begin{array}{l}{[14],} \\
{[11],} \\
{[16]}\end{array}$ \\
\hline 8. & $\begin{array}{c}\text { Financial } \\
\text { Concerns } \\
\text { (FC) }\end{array}$ & $\begin{array}{l}\text { This includes lack of } \\
\text { capital and finance for } \\
\text { starting an enterprise or to } \\
\text { continue the existing } \\
\text { enterprise. }\end{array}$ & $\begin{array}{c}16],[13], \\
{[16],[6],} \\
{[7],[15],} \\
{[9],[18],} \\
{[19],[20],} \\
{[22],[23]}\end{array}$ \\
\hline 9. & $\begin{array}{l}\text { Marketing } \\
\text { Issues } \\
\text { (MI) }\end{array}$ & $\begin{array}{l}\text { This includes marketing } \\
\text { issues, severe market } \\
\text { regulations, cut throat } \\
\text { competition for survival. }\end{array}$ & {$[7],[8],[6]$} \\
\hline 10. & Geographical & $\begin{array}{l}\text { This includes barriers such } \\
\text { as geographical location of }\end{array}$ & [21] \\
\hline
\end{tabular}

\begin{tabular}{|c|c|c|c|}
\hline & $\begin{array}{c}\text { factors } \\
(\mathrm{GF})\end{array}$ & $\begin{array}{c}\text { enterprise, communication } \\
\text { problems and commutation } \\
\text { issues. }\end{array}$ & \\
\hline 11. & $\begin{array}{c}\text { Political } \\
\text { factors } \\
(\mathrm{PF})\end{array}$ & $\begin{array}{c}\text { This includes corruption, } \\
\text { political instability and } \\
\text { inadequate legal system . }\end{array}$ & {$[3],[7]$} \\
\hline 12. & Lack & $\begin{array}{c}\text { This includes absence of } \\
\text { training towards women } \\
\text { entrepreneurship, } \\
\text { of } \\
\text { of gelopment programmes }\end{array}$ & {$[14]$,} \\
& training & {$[24]$} \\
(LT) & & \\
\hline
\end{tabular}

Warfield [25] proposed the ISM technique in 1994. Following the process results in creating a structured graph from the set of unique interrelated variables. The process goes through the various steps viz. identifying the relevant elements and establishing a contextual relationship amongst them; then an Structural self- interaction matrix is developed to establish the lead to relationship amongst the two variables $i \& j$. An initial reachability matrix is then created which eventually leads to the development of final reachability matrix and thereafter reachability set and antecedent set for each criterion. In every iteration a top level element is selected for which the reachability set and intersection sets are the same.

\section{DEVELOPMENT OF ISM MODEL}

In this section, ISM model is developed for studying the interrelationships amongst the various barriers to women entrepreneurship in India. Out of the total variables, 12 important criteria are considered viz. Lack of motivation (LM), Lack of education \& training (LET), Family problems (FP), Lack of infrastructure (LIF), Negative attitude of society (NAS), Lack of knowledge and awareness (LKA), Absence of societal concerns (ASC), Financial concerns (FC), Market issues (MI), Geographical factors (GF), Political factors (PF), Peer group factor (PGF).

\subsection{Construction of Structural Self - Interaction Matrix (SSIM)}

This matrix gives the pair-wise relationship between two variables i.e. $i$ and $j$ based on VAXO. SSIM has been presented below in Fig 1.

Explanation : Lack of education, family problems, negative attitude of society, lack of knowledge and awareness, absence of societal concerns, market issues, absence of peer group influence all contribute to lack in motivating to take up entrepreneurship as a career for women. Similarly family problems, negative attitude of society, financial concerns restricts women to take up higher education. Family problems could be the cause of lack of appropriate infrastructure, lack of education, lack of capital and lack of training as well as choosing the location for enterprise. Non co-operation from society, government authorities as well as organizations, lack of capital, corruption and also to some extent lack of knowledge and awareness and appropriate training results in poor infrastructure. Poor infrastructure will affect the growth of enterprise as it will hinder the process of timely delivery and communication to clients. Also geographical location and issues are related with societal issues and vice versa. For example in metro cities, opportunities for entrepreneurial growth could be more as government schemes and 
organizational support could be received. In remote or rural areas, positive government support and society attitude is hard to obtain. But at the same time financial crunch could be a major hurdle to survive as these areas are costly to accommodate. Absence of any role model makes it difficult to change the negative attitude of society towards women entrepreneurship. Also non co-operation from society and organizational support creates financial crunch or lack of capital issues. Also absence of peer group influence and political stagnation creates financial hurdles for a successful women entrepreneurship. In remote areas, it is hard to arrange for funds as opportunities are less and people are dubious to lend you financial help. Lack of knowledge and awareness may create market issues and also lack of training as the person is not adequately aware of how to create successful market of its product. This could also be due to lack of education, financial constraints and family constraints. In India, women are mostly confined to the four walls of house. Hence receptivity of knowledge and external exposure is the minimum. To run an enterprise, you are required to communicate effectively in public, to the clients. Also political constraints and corruption hinders the successful sale of product in potential market

\subsection{Construction of Initial Reachability Matrix and final reachability matrix}

The SSIM has been converted in to a binary matrix called the initial reachability matrix shown in fig. 2 by substituting V, A, $\mathrm{X}, \mathrm{O}$ by 1 or 0 as per the case. After incorporating the transitivity, the final reachability matrix is shown below in the Fig 3.

\begin{tabular}{|c|c|c|c|c|c|c|c|c|c|c|c|c|c|}
\hline & $\begin{array}{c}\text { Barrier } \\
\text { s }\end{array}$ & 1 & 2 & 3 & 4 & 5 & 6 & 7 & 8 & 9 & 10 & 11 & 12 \\
\hline & & LM & LE & FP & LI & NAS & LKA & ASC & FC & MI & GF & PF & LT \\
\hline 1 & LM & & A & A & A & A & A & A & A & A & A & A & A \\
\hline 2 & LE & & & A & V & A & O & A & A & V & O & O & V \\
\hline 3 & FP & & & & V & O & V & O & X & O & V & A & V \\
\hline 4 & LI & & & & & A & A & A & A & O & A & A & A \\
\hline 5 & NAS & & & & & & O & V & V & V & O & V & V \\
\hline 6 & LKA & & & & & & & O & A & V & A & A & V \\
\hline 7 & ASC & & & & & & & & V & V & A & A & V \\
\hline 8 & FC & & & & & & & & & V & X & A & V \\
\hline 9 & MI & & & & & & & & & A & A & A \\
\hline 10 & GF & & & & & & & & & & A & V \\
\hline 11 & PF & & & & & & & & & & & & V \\
\hline 12 & LT & & & & & & & & & & & \\
\hline
\end{tabular}

Fig 1: SSIM matrix for pair wise relationship amongst barriers

\begin{tabular}{|c|c|c|c|c|c|c|c|c|c|c|c|c|c|}
\hline & $\begin{array}{c}\text { Barrier } \\
\text { s }\end{array}$ & 1 & 2 & 3 & 4 & 5 & 6 & 7 & 8 & 9 & 10 & 11 & 12 \\
\hline & & LM & LE & FP & LI & NAS & LKA & ASC & FC & MI & GF & PF & LT \\
\hline 1 & LM & 1 & 0 & 0 & 0 & 0 & 0 & 0 & 0 & 0 & 0 & 0 & 0 \\
\hline 2 & LE & 1 & 1 & 0 & 1 & 0 & 0 & 0 & 0 & 1 & 0 & 0 & 1 \\
\hline 3 & FP & 1 & 1 & 1 & 1 & 0 & 1 & 0 & 1 & 0 & 1 & 0 & 1 \\
\hline 4 & LI & 1 & 0 & 0 & 1 & 0 & 0 & 0 & 0 & 0 & 0 & 0 & 0 \\
\hline 5 & NAS & 1 & 1 & 0 & 1 & 1 & 1 & 1 & 1 & 1 & 0 & 1 & 1 \\
\hline 6 & LKA & 1 & 0 & 0 & 1 & 0 & 1 & 0 & 0 & 1 & 0 & 0 & 1 \\
\hline 7 & ASC & 1 & 1 & 1 & 0 & 0 & 0 & 1 & 1 & 1 & 0 & 0 & 1 \\
\hline 8 & FC & 1 & 1 & 1 & 1 & 0 & 1 & 0 & 1 & 1 & 1 & 0 & 1 \\
\hline 9 & MI & 1 & 0 & 0 & 0 & 0 & 0 & 0 & 0 & 1 & 0 & 0 & 0 \\
\hline 10 & GF & 1 & 0 & 0 & 1 & 0 & 1 & 1 & 1 & 1 & 1 & 0 & 1 \\
\hline 11 & PF & 1 & 0 & 1 & 1 & 0 & 1 & 1 & 1 & 1 & 1 & 1 & 1 \\
\hline 12 & LT & 1 & 0 & 0 & 1 & 0 & 0 & 0 & 0 & 1 & 0 & 0 & 1 \\
\hline
\end{tabular}

Fig 2: Initial reachability matrix 


\begin{tabular}{|c|c|c|c|c|c|c|c|c|c|c|c|c|c|c|}
\hline & Barriers & 1 & 2 & 3 & 4 & 5 & 6 & 7 & 8 & 9 & 10 & 11 & 12 & \\
\hline & & LM & LE & FP & LI & NAS & LKA & ASC & FC & MI & GF & PF & LT & D.P \\
\hline 1 & LM & 1 & 0 & 0 & 0 & 0 & 0 & 0 & 0 & 0 & 0 & 0 & 0 & 10 \\
\hline 2 & LE & 1 & 1 & 0 & 1 & 0 & 0 & 0 & 0 & 1 & 0 & 0 & 1 & 9 \\
\hline 3 & FP & 1 & 1 & 1 & 1 & 0 & 1 & 0 & 1 & 0 & 1 & 0 & 1 & 12 \\
\hline 4 & LI & 1 & 0 & 0 & 1 & 0 & 0 & 0 & 0 & 0 & 0 & 0 & 0 & 4 \\
\hline 5 & NAS & 1 & 1 & 0 & 1 & 1 & 1 & 1 & 1 & 1 & 0 & 1 & 1 & 1 \\
\hline 6 & LKA & 1 & 0 & 0 & 1 & 0 & 1 & 0 & 0 & 1 & 0 & 0 & 1 & 13 \\
\hline 7 & ASC & 1 & 1 & 1 & 0 & 0 & 0 & 1 & 1 & 1 & 0 & 0 & 1 & 10 \\
\hline 8 & FC & 1 & 1 & 1 & 1 & 0 & 1 & 0 & 1 & 1 & 1 & 0 & 1 & 6 \\
\hline 9 & MI & 1 & 0 & 0 & 0 & 0 & 0 & 0 & 0 & 1 & 0 & 0 & 0 & 6 \\
\hline 10 & GF & 1 & 0 & 0 & 1 & 0 & 1 & 1 & 1 & 1 & 1 & 0 & 1 & 3 \\
\hline 11 & PF & 1 & 0 & 1 & 1 & 0 & 1 & 1 & 1 & 1 & 1 & 1 & 1 & 3 \\
\hline 12 & LT & 1 & 0 & 0 & 1 & 0 & 0 & 0 & 0 & 1 & 0 & 0 & 1 & 6 \\
\hline & De..P & 6 & 4 & 7 & 8 & 14 & 3 & 5 & 7 & 8 & 7 & 12 & 7 & \\
\hline
\end{tabular}

Fig 3 : Final reachability matrix

D.P : Driving power ; De.P : dependence power

\subsection{Level Partition}

From the final reachability matrix, reachability and final antecedent set for each factor are found. The element for which the reachability and intersection sets are same are the top-level element in the ISM hierarchy. After the identification of top level element, it is separated out from the other elements and the process continues for next level of elements. Iterations have been shown from table 3 - table 13 below .

Table 3 : Iteration I

\begin{tabular}{|c|c|c|c|c|}
\hline $\begin{array}{l}\text { Sr. } \\
\text { No. }\end{array}$ & $\begin{array}{c}\text { Reachability } \\
\text { set }\end{array}$ & Antecedent set & $\begin{array}{c}\text { Intersection } \\
\text { set }\end{array}$ & $\begin{array}{l}\text { Itera } \\
\text { tion }\end{array}$ \\
\hline 1 & 1 & $\begin{array}{c}1,2,3,4,5,6,7,8,9 \\
10,11,12\end{array}$ & 1 & \\
\hline 2 & 1,9 & $\begin{array}{c}2,3,5,6,7,8,9,10,1 \\
1,12\end{array}$ & 9 & \\
\hline 3 & $1,4,9$ & $\begin{array}{c}2,3,4,5,6,8,10 \\
11,12\end{array}$ & 4 & I \\
\hline 4 & $\begin{array}{c}1,2,3,6,7,8,9 \\
10,12\end{array}$ & $3,5,7$ & 3,7 & \\
\hline 5 & $1,4,9,12$ & $\begin{array}{c}2,3,5,6,8,10,11,1 \\
2\end{array}$ & 13 & \\
\hline 6 & $\begin{array}{c}1,4,9,13,10 \\
8,6,2\end{array}$ & $3,5,7,10$ & 10 & \\
\hline 7 & 3,7 & $3,5,7,11,12$ & 3,7 & \\
\hline 8 & $8,10,12$ & $3,5,7,8,10,11$ & 8,10 & \\
\hline 9 & 2,12 & $2,3,5,7,8,10,11$ & 2 & \\
\hline 10 & 6,12 & $3,5,6,7,8,10,11$ & 6 & \\
\hline 11 & $1,2,3,4,5,6$ & 5 & 5 & \\
\hline
\end{tabular}

\begin{tabular}{|c|c|c|c|}
\hline & $7,8,9,10,11,12$ & & \\
\hline 12 & $\begin{array}{c}1,2,4,8,9,10, \\
11,13\end{array}$ & 11 & 11 \\
\hline 13 & $3,7,2,6,8,10$ & $3,5,7,8$ & $3,7,8$ \\
\hline 14 & $3,5,7$ & 5 & 5 \\
\hline 15 & 11 & 5,11 & 11 \\
\hline
\end{tabular}

Table 4 : Iteration II

\begin{tabular}{|c|c|c|c|c|}
\hline $\begin{array}{l}\text { Sr. } \\
\text { No. }\end{array}$ & $\begin{array}{c}\text { Reachability } \\
\text { set }\end{array}$ & $\begin{array}{c}\text { Antecedent } \\
\text { set }\end{array}$ & $\begin{array}{c}\text { Intersection } \\
\text { set }\end{array}$ & $\begin{array}{l}\text { Itera } \\
\text { tion }\end{array}$ \\
\hline 2 & 9 & $\begin{array}{c}2,3,4,5,6,7,8 \\
9,10,11,12\end{array}$ & 9 & \multirow{9}{*}{ II } \\
\hline 3 & 4,9 & $\begin{array}{c}2,3,4,5,6,8 \\
10,11,12\end{array}$ & 4 & \\
\hline 4 & $\begin{array}{c}2,3,6,7,8,9,10 \\
12\end{array}$ & $3,5,7$ & 3,7 & \\
\hline 5 & $4,9,12$ & $\begin{array}{c}2,3,5,6,8.10 \\
11,12\end{array}$ & 13 & \\
\hline 6 & $4,9,12,10,8,6,2$ & $3,5,7,10$ & 10 & \\
\hline 7 & 3,7 & $3,5,7,11,12$ & 3,7 & \\
\hline 8 & $8,10,12$ & $\begin{array}{c}3,5,7,8,10 \\
11\end{array}$ & 8,10 & \\
\hline 9 & 2,12 & $\begin{array}{c}2,3,5,7,8,10 \\
11\end{array}$ & 2 & \\
\hline 10 & 6,12 & $\begin{array}{c}3,5,6,7,8,10 \\
11\end{array}$ & 6 & \\
\hline
\end{tabular}




\begin{tabular}{|c|c|c|c|}
\hline 11 & $\begin{array}{c}2,3,4,5,6,7,8,9, \\
10,11,12\end{array}$ & 5 & 5 \\
\cline { 2 - 4 } 12 & $\begin{array}{c}2,4,8,9,10,11,1 \\
2\end{array}$ & 11 & 11 \\
\cline { 1 - 3 } 13 & $3,7,2,6,8,10$ & $3,5,7,8$ & $3,7,8$ \\
\hline 14 & $3,7,5$ & 5 & 5 \\
\hline 15 & 11 & 5,11 & 11 \\
\hline
\end{tabular}

\begin{tabular}{|c|c|c|c|}
\hline 11 & $2,8,10,11,12$ & 11 & 11 \\
\hline 12 & $3,7,2,6,8,10$ & $3,5,7,8$ & $3,7,8$ \\
\hline 13 & $3,5,7$ & 5 & 5 \\
\hline 14 & 11 & 5,11 & 11 \\
\hline
\end{tabular}

Table 5 : Iteration III

\begin{tabular}{|c|c|c|c|c|}
\hline $\begin{array}{l}\text { Sr. } \\
\text { No. }\end{array}$ & $\begin{array}{c}\text { Reachability } \\
\text { set }\end{array}$ & $\begin{array}{c}\text { Antecedent } \\
\text { set }\end{array}$ & $\begin{array}{c}\text { Intersection } \\
\text { set }\end{array}$ & $\begin{array}{c}\text { Itera } \\
\text { tion }\end{array}$ \\
\hline 2 & 4 & $\begin{array}{c}2,3,4,5,6,8,10 \\
11,12\end{array}$ & 4 & \multirow{13}{*}{ III } \\
\hline 3 & $\begin{array}{c}2,3,6,7,8,10 \\
12\end{array}$ & $3,5,7$ & 3,7 & \\
\hline 4 & 4,12 & $\begin{array}{c}2,3,5,6,8.10 \\
11,12\end{array}$ & 12 & \\
\hline 5 & $4,12,10,8,6,2$ & $3,5,7,10$ & 10 & \\
\hline 6 & 3,7 & $3,5,7,11,12$ & 3,7 & \\
\hline 7 & $8,10,12$ & $3,5,7,8,10,11$ & 8,10 & \\
\hline 8 & 2,12 & $\begin{array}{c}2,3,5,7,8,10 \\
11\end{array}$ & 2 & \\
\hline 9 & 6,12 & $\begin{array}{c}3,5,6,7,8,10 \\
11\end{array}$ & 6 & \\
\hline 10 & $\begin{array}{c}2,3,4,5,6,7,8 \\
10,11,12\end{array}$ & 5 & 5 & \\
\hline 11 & $2,4,8,10,11,12$ & 11 & 11 & \\
\hline 12 & $3,7,2,6,8,10$ & $3,5,7,8$ & $3,7,8$ & \\
\hline 13 & $3,5,7$ & 5 & 5 & \\
\hline 14 & 11 & 5,11 & 11 & \\
\hline
\end{tabular}

Table 6 : Iteration IV

\begin{tabular}{|c|c|c|c|c|}
\hline $\begin{array}{l}\text { Sr. } \\
\text { No. }\end{array}$ & $\begin{array}{c}\text { Reachability } \\
\text { set }\end{array}$ & Antecedent set & $\begin{array}{c}\text { Intersectio } \\
\text { n set }\end{array}$ & $\begin{array}{l}\text { Itera } \\
\text { tion }\end{array}$ \\
\hline 3 & $\begin{array}{c}2,3,6,7,8,10 \\
12\end{array}$ & $3,5,7$ & 3,7 & \multirow{8}{*}{ IV } \\
\hline 4 & 12 & $2,3,5,6,8.10,11,12$ & 12 & \\
\hline 5 & $12,10,8,6,2$ & $3,5,7,10$ & 10 & \\
\hline 6 & 3,7 & $3,5,7,11,12$ & 3,7 & \\
\hline 7 & $8,10,12$ & $3,5,7,8,10,11$ & 8,10 & \\
\hline 8 & 2,12 & $2,3,5,7,8,10,11$ & 2 & \\
\hline 9 & 6,12 & $3,5,6,7,8,10,11$ & 6 & \\
\hline 10 & $\begin{array}{c}2,3,4,5,6,7,8 \\
10,11,12\end{array}$ & 5 & 5 & \\
\hline
\end{tabular}

Table 7 : Iteration $\mathrm{V}$

\begin{tabular}{|c|c|c|c|c|}
\hline $\begin{array}{l}\text { Sr. } \\
\text { No. }\end{array}$ & $\begin{array}{c}\text { Reachability } \\
\text { set }\end{array}$ & $\begin{array}{c}\text { Antecedent } \\
\text { set }\end{array}$ & $\begin{array}{c}\text { Intersection } \\
\text { set }\end{array}$ & $\begin{array}{c}\text { Itera } \\
\text { tion }\end{array}$ \\
\hline 3 & $2,3,6,7,8,10$ & $3,5,7$ & 3,7 & \\
\hline 5 & $10,8,6,2$ & $3,5,7,10$ & 10 & \\
\hline 6 & 3,7 & $3,5,7,11$ & 3,7 & \\
\hline 7 & 8,10 & $\begin{array}{c}3,5,7,8,10, \\
11\end{array}$ & 8,10 & V \\
\hline 8 & 2 & $\begin{array}{c}2,3,5,7,8,10 \\
11\end{array}$ & 2 & \\
\hline 9 & 6 & $\begin{array}{c}3,5,6,7,8,10 \\
11\end{array}$ & 6 & \\
\hline 10 & $\begin{array}{c}2,3,4,5,6,7,8 \\
10,11\end{array}$ & 5 & 5 & \\
\hline 11 & $2,8,10,11$ & 11 & 11 & \\
\hline 12 & $3,7,2,6,8,10$ & $3,5,7,8$ & $3,7,8$ & \\
\hline 13 & $3,5,7$ & 5 & 5 & \\
\hline 14 & 11 & 5,11 & 11 & \\
\hline
\end{tabular}

Table 8 : Iteration VI

\begin{tabular}{|c|c|c|c|c|}
\hline $\begin{array}{l}\text { Sr. } \\
\text { No. }\end{array}$ & $\begin{array}{c}\text { Reachability } \\
\text { set }\end{array}$ & $\begin{array}{c}\text { Antecedent } \\
\text { set }\end{array}$ & $\begin{array}{c}\text { Intersection } \\
\text { set }\end{array}$ & $\begin{array}{l}\text { Itera } \\
\text { tion }\end{array}$ \\
\hline 3 & $2,3,6,7,8,10$ & $3,5,7$ & 3,7 & \multirow{9}{*}{ VI } \\
\hline 5 & $10,8,6,2$ & $3,5,7,10$ & 10 & \\
\hline 6 & 3,7 & $3,5,7,11$ & 3,7 & \\
\hline 7 & 8,10 & $3,5,7,8,10,11$ & 8,10 & \\
\hline 10 & $\begin{array}{c}2,3,4,5,6,7,8 \\
10,11\end{array}$ & 5 & 5 & \\
\hline 11 & $2,8,10,11$ & 11 & 11 & \\
\hline 12 & $3,7,2,6,8,10$ & $3,5,7,8$ & $3,7,8$ & \\
\hline 13 & $3,5,7$ & 5 & 5 & \\
\hline 14 & 11 & 5,11 & 11 & \\
\hline
\end{tabular}


Table 9 : Iteration VII

\begin{tabular}{|c|c|c|c|c|}
\hline $\begin{array}{c}\text { Sr. } \\
\text { No. }\end{array}$ & $\begin{array}{c}\text { Reachability } \\
\text { set }\end{array}$ & $\begin{array}{c}\text { Antecedent } \\
\text { set }\end{array}$ & $\begin{array}{c}\text { Intersection } \\
\text { set }\end{array}$ & $\begin{array}{c}\text { Itera } \\
\text { tion }\end{array}$ \\
\hline 3 & 3,7 & $3,5,7$ & 3,7 & \\
\cline { 1 - 3 } & $\mathbf{3 , 7}$ & $3,5,7,11,12$ & 3,7 & \multirow{2}{*}{ VII } \\
\hline 10 & $3,4,5,7,11$ & 5 & 5 & \\
\hline 11 & 2,11 & 11 & 11 & \\
\hline 12 & 3,7 & $3,5,7$ & 3,7 & \\
\hline 13 & $3,5,7$ & 5 & 5 & \\
\hline 14 & 11 & 5,11 & 11 & \\
\hline
\end{tabular}

Table 10 : Iteration VIII

\begin{tabular}{|c|c|c|c|c|}
\hline $\begin{array}{c}\text { Sr. } \\
\text { No. }\end{array}$ & $\begin{array}{c}\text { Reachability } \\
\text { set }\end{array}$ & $\begin{array}{c}\text { Antecedent } \\
\text { set }\end{array}$ & $\begin{array}{c}\text { Intersection } \\
\text { set }\end{array}$ & $\begin{array}{c}\text { Itera } \\
\text { tion }\end{array}$ \\
\hline 10 & 5,11 & 5 & 5 & \multirow{2}{*}{ VIII } \\
\hline 11 & $\mathbf{1 1}$ & 5,11 & 11 & \\
\hline 13 & 5 & 5 & 5 & \\
\hline 14 & 11 & 5,11 & 11 & \\
\hline
\end{tabular}

Table 11 : Iteration IX

\begin{tabular}{|c|c|c|c|c|}
\hline $\begin{array}{c}\text { Sr. } \\
\text { No. }\end{array}$ & $\begin{array}{c}\text { Reachability } \\
\text { set }\end{array}$ & $\begin{array}{c}\text { Antecedent } \\
\text { set }\end{array}$ & $\begin{array}{c}\text { Intersection } \\
\text { set }\end{array}$ & $\begin{array}{c}\text { Itera } \\
\text { tion }\end{array}$ \\
\hline 10 & 5 & 5 & 5 & \multirow{2}{*}{ IX } \\
\hline 13 & $\mathbf{5}$ & 5 & $\mathbf{5}$ & \\
\hline
\end{tabular}

\subsection{Classification of factors}

Fig. 4 below shows the driving power and dominance diagram.

Driving Power \& Dominance Diagram (MICMAC analysis)

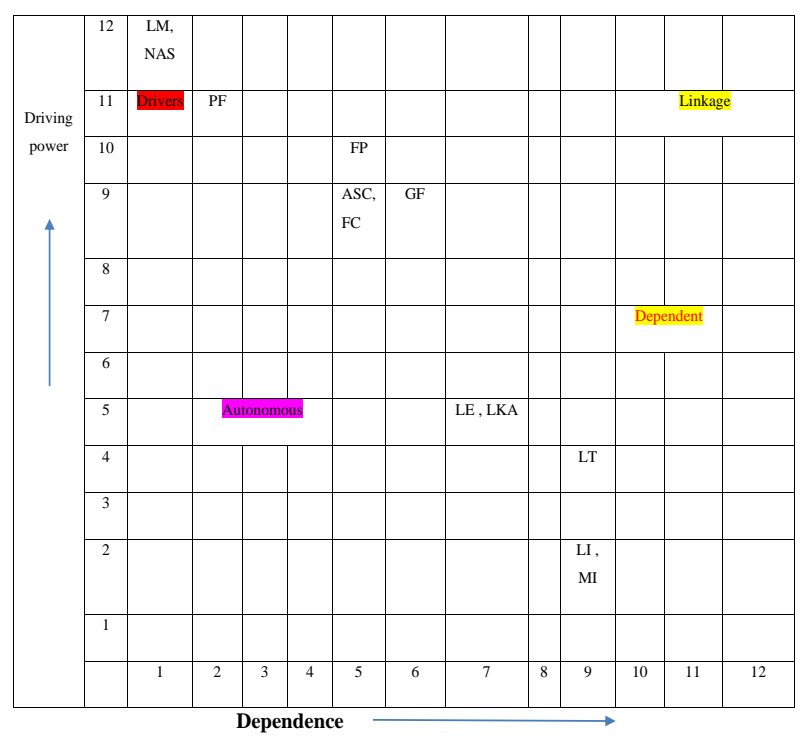

Fig . 4: Driving power and dependence diagram

\subsection{ISM model}

An ISM model is developed ( as shown in fig. 5 below ) after arranging the elements as per their interaction or dependence relationships.

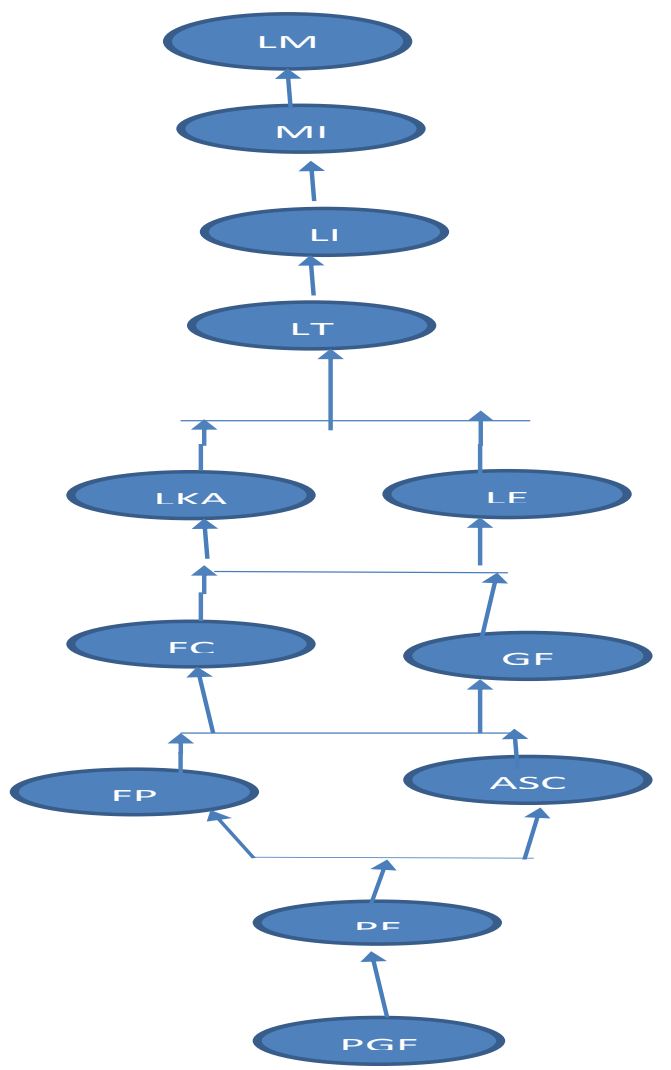

Fig 5 : ISM Model

\section{MANAGERIAL IMPLICATIONS \& CONCLUSIONS}

The purpose of this study is to specifically identify the key barriers to the creation of a small business among Indian women entrepreneurs. This study would benefit academicians and research scholars with its useful insights. Women entrepreneurs, scholars and policymakers will gain greater understanding through this causal framework of barriers. Knowledge and containment of these barriers will help in fostering a more encouraging environment to attempt to women entrepreneurship.

\section{ACKNOWLEDGMENTS}

Our thanks to the anonymous reviewers whose comments have helped us in improving the manuscript .

\section{REFERENCES}

[1] Mitchelmore, S. \& Rowley, J. 2013. Entrepreneurial competencies of women entrepreneurs pursuing business growth. Journal of Small Business and Enterprise Development. 20, 125-142.

[2] Dana, L.P. 2000. Creating entrepreneurs in India', Journal of Small Business Management . 38(1), 86-91.

[3] Hatala, J.P.2005 . Identifying barriers to selfemployment: the development and validation of the barriers to entrepreneurship success tool', Performance Improvement Quarterly. 18(4), 50-70.

[4] Choo, S. and Wong, M. 2006. Entrepreneurial intention: triggers and barriers to new venture creations in 
Singapore, Singapore Management Review, 28(2), 4766.

[5] Goyal, M. and Prakash, J.2011. Women entrepreneurs in India-Problems and Prospects" International Journal of Multidisciplinary Research, 1(5).

[6] Hooda, N. 2017. Women Entrepreneurship: Genesis For Successful Business Model. International Journal of Management, 8 (4), 31-39.

[7] Raeesi1, R. , Dastranj, M. Mohammadi1, S. and Rasouli, E. 2013. Understanding the Interactions among the Barriers to Entrepreneurship Using Interpretive Structural Modeling, International Journal of Business and Management 8(13), ISSN 1833-3850.

[8] Vijayaragavan, T. 2014. Problems and Opportunities of Women Entrepreneurs in India. International Journal of Business and Management Invention . 3(7) , 59-61.

[9] Sugaraj, J.M. and Salve P.S .2014. A study of women Entrepreneurship and their problems in the Development in Western Maharashtra. ISOR Journal of Economics and Finance, 3.

[10] Tripathi, R. and Singh, V.P. 2017. Women Entrepreneurship In Developing Countries. International Journal of Management, 8 (4), 1-7.

[11] Ghosh, P. and Cheruvalath, R. 2007. Indian female entrepreneurs as catalysts for economic growth and development. The International Journal of Entrepreneurship and Innovation, 8(2), 139-148.

[12] Mehta, A., \& Mehta, M. C. 2011. Rural women entrepreneurship in India: opportunities and challenges. International Conference on Humanities, Geography and Economics (ICHGE'2011).

[13] Raghuvanshi , J., Agrawal , R. and Ghosh , P.K. 2017. Analysis of Barriers to Women Entrepreneurship: The DEMATEL Approach . Journal of Entrepreneurship 26(2), 220-238.

[14] Javaheri, F. and Ghozati, S. 2004. Barriers to women's entrepreneurship; Influence of gender inequality on women entrepreneurship in Iran. Journal of Sociology, 5(2), 161- 178.

[15] Veena M., and Nagaraj, N. 2014. A study on Problems Faced by Women Entrepreneurs in Mysore District .
International Journal of Engineering and Management Research, 4(1), 45-50.

[16] Iakovleva, T., Solesvik, M. \& Trifilova, A. 2013. Financial availability and government support for women entrepreneurs in transitional economies: The case of Russia and Ukraine. Journal of Small Business and Enterprise Development, 20, 314-340.

[17] Tuschano, J. W. 2013. Women entrepreneurs in unorganised sector: a case study of Vasai taluka. (Unpublished Ph.D. thesis), S.N.D.T. Women's University, Mumbai, India.

[18] Meyer, N. and Mostert, C. 2016. Perceived Barriers And Success Factors Of Female Entrepreneurs Enrolled In An Entrepreneurial Programme, International Journal of Social Sciences and humanity studies, 8(1). ISSN: 1309-8063.

[19] Gowramma A.A , P. and Kumar, S. R. 2018. Challenges Of Women Entrepreneurs - Case Study With Special Reference To Sme Industries In Coorg, Karnataka. International Journal of Mechanical Engineering and Technology (IJMET), 9(1), 266-275.

[20] Umamaheswari, V. 2011. A study on the development of women entrepreneurs in Dindigul district. (Un-Published $\mathrm{Ph}$. D Thesis), Mother Teresa Women's University, Tamil Nadu, India.

[21] Rani J, Sinha , S.K. 2016. Barriers Facing Women Entrepreneurs in Rural India: A Study in Haryana. Amity Journal of Entrepreneurship,1(1), 86-100.

[22] Huarng, K., Mas-Tur, A. \& Yu, T.H. 2012. Factors affecting the success of women entrepreneurs. International Entrepreneurship and Management Journal. $8,487-497$.

[23] Devi, B.P. 2014. Problems and Prospects of Women Entrepreneurship in India. International Journal of Science and Research . 3(9) .

[24] Golrad, P. 2007. Attributes, motives and goals to encourage women to start business. Quarterly Journal of Business. 44, 267-295.

[25] Warfield, J. N. 1974. Developing interconnection matrices in structural modeling. IEEE Transactions on System, Man, and Cybernetics, SMC-4 (1), 81-87. 